\title{
Dissecting Long-Term Adjustments of Photoprotective and Photo-Oxidative Stress Acclimation Occurring in Dynamic Light Environments
}

\author{
Shizue Matsubara ${ }^{1}$, Trang Schneider ${ }^{1,2}$ and Veronica G. Maurino ${ }^{3 *}$ \\ ${ }^{1}$ IBG-2: Plant Sciences, Institute of Bio- and Geosciences, Forschungszentrum Jülich, Jülich, Germany, ${ }^{2}$ iGRAD-Plant, \\ Heinrich-Heine-Universität, Düsseldorf, Germany, ${ }^{3}$ Institute of Developmental and Molecular Biology of Plants, Plant \\ Molecular Physiology and Biotechnology Group, Heinrich-Heine-Universität and Cluster of Excellence on Plant Sciences, \\ Düsseldorf, Germany
}

OPEN ACCESS

Edited by:

Massimiliano Tattini,

National Research Council, Italy

Reviewed by:

Or Sperling,

Agricultural Research Organization,

Volcani Center, Israel

Lucia Guidi,

Università di Pisa, Italy

*Correspondence:

Veronica G. Maurino

veronica.maurino@uni-duesseldorf.de

Specialty section:

This article was submitted to

Functional Plant Ecology,

a section of the journal

Frontiers in Plant Science

Received: 12 July 2016 Accepted: 26 October 2016 Published: 09 November 2016

Citation:

Matsubara S, Schneider T and Maurino VG (2016) Dissecting Long-Term Adjustments

of Photoprotective

and Photo-Oxidative Stress Acclimation Occurring in Dynamic

Light Environments.

Front. Plant Sci. 7:1690.

doi: 10.3389/fpls.2016.01690
Changes in light intensity directly affect the performance of the photosynthetic apparatus. Light energy absorbed in excess of cells' needs leads to production of reactive oxygen species and photo-oxidative damage. Excess light in both constant and dynamic environments induces photoprotective acclimation in plants. Distinct sets of signals and regulatory mechanisms are involved in acclimatory adjustment of photoprotection and photosynthesis under constant and dynamic (fluctuating) light conditions. We are still far away from drawing a comprehensive picture of acclimatory signal transduction pathways, particularly in dynamic environments. In this perspective article, we propose the use of Arabidopsis plants that produce $\mathrm{H}_{2} \mathrm{O}_{2}$ in chloroplasts (GO plants) under atmospheric $\mathrm{CO}_{2}$ levels as a tool to study the mechanisms of long-term acclimation to photo-oxidative stress. In our opinion there are new avenues to future investigations on acclimatory adjustments and signal transduction occurring in plants under dynamic light environments.

Keywords: acclimation, fluctuating light, photoprotection, reactive oxygen species, retrograde signaling

\section{ACCLIMATION TO PHOTO-OXIDATIVE STRESS IS INDUCED BY FLUCTUATING LIGHT}

Rapid climate changes and transformation of landscapes by extensive agricultural practices impose environmental perturbations. Plants in the affected areas respond to the perturbations through acclimation (within generation) or adaptation (over generations). Light intensity can vary rapidly by a few orders of magnitude as clouds travel in the sky or wind moves outer canopy leaves and taller plants. Especially, wind can briefly expose inner canopy leaves and understory plants to intense sunlight. Upon large and abrupt increase in light intensity, photosynthetic light energy utilization is limited biochemically. This is attributed to low availability of the Calvin-Benson cycle intermediates, low activation state of Ribulose-1,5-bisphosphate carboxylase/oxygenase (RubisCO), and also low stomatal conductance measured in leaves under low light (LL) conditions (Kirschbaum and Pearcy, 1988; Pearcy, 1990).

When light energy is absorbed by photosynthetic pigments in excess of cells' needs for reducing equivalents and chemical energy (excess light, EL), it can lead to production of reactive 
oxygen species (ROS) and photo-oxidative damage in oxygenic photosynthetic organisms. A range of mechanisms have evolved to reduce uncontrolled production of ROS and to protect the photosynthetic apparatus against their detrimental effects (Foyer and Noctor, 2000; Endo and Asada, 2008; Li et al., 2009). These include thermal energy dissipation which is rapidly induced in light-harvesting antenna complexes by a proton concentration gradient $(\Delta \mathrm{pH})$ across the thylakoid membrane (termed $\mathrm{qE}$ ), alternative sinks for excess electrons (e.g., water-water cycle and cyclic electron flows around photosystem I, CEF) which contribute to $\Delta \mathrm{pH}$ formation especially when liner electron transport rate (ETR) is low, and enzymatic and non-enzymatic antioxidative systems which detoxify ROS (Figure 1A).

When EL conditions persist, plants are able to augment their photoprotective capacities via long-term acclimation. In general, multiple mechanisms of photoprotective acclimation (Figure 1A) operate in plants under constant as well as dynamic EL environments. In particular, fluctuating light (FL) with short periods of EL (i.e., dynamic EL) induces, primarily or initially, long-term acclimatory changes that are characterized by improved protection against photo-oxidative stress and reduced carbon gain (Alter et al., 2012). For instance, LL-grown Arabidopsis plants upregulate photoprotection in highly dynamic EL conditions without developing symptoms of severe photooxidative injuries, such as strong photoinhibition or bleaching (Alter et al., 2012). Similar photoprotective responses are also seen during acclimation to high light (HL, i.e., constant EL), although in HL they are often accompanied by enhancement of photosynthesis and thus increased carbon gain (Leakey et al., 2002; Alter et al., 2012). There seems to be an inverse relationship between the maximum photosynthetic capacity, which is developed through photosynthetic acclimation, and the frequency of LL-HL transitions (Retkute et al., 2015). Selective upregulation of photoprotection, but not photosynthesis, in highly dynamic FL suggests that distinct sets of signals and regulatory mechanisms are involved in acclimatory adjustment of photoprotection and photosynthesis, and that signal molecules, which trigger photo-oxidative stress acclimation, are produced in leaves under the FL conditions. In this article we use the term "FL" to refer to highly dynamic EL conditions, while we are aware that FL may not always cause EL and photo-oxidative stress, depending on the amplitude and frequency of light fluctuations (Yin and Johnson, 2000; Alter et al., 2012; Retkute et al., 2015).

\section{CHLOROPLAST RETROGRADE SIGNALING IS INVOLVED IN PHOTO-OXIDATIVE STRESS ACCLIMATION}

Today it is widely recognized that ROS not only can damage cellular components but also act as signals to induce abiotic and biotic stress responses (Foyer and Noctor, 2000; Apel and Hirt, 2004; Mittler et al., 2011; Karpinski et al., 2013; Dietz et al., 2016). Multiple ROS can be generated in chloroplasts under photooxidative stress, such as singlet oxygen $\left({ }^{1} \mathrm{O}_{2}\right)$, superoxide anion radical $\left(\mathrm{O}_{2}^{\bullet-}\right)$, hydroxyl radical $(\mathrm{OH})$, and hydrogen peroxide $\left(\mathrm{H}_{2} \mathrm{O}_{2}\right)$ (Asada, 1999; Foyer and Noctor, 2000). Among these, $\mathrm{H}_{2} \mathrm{O}_{2}$ alone would be able to diffuse into the cytosol because high reactivity and charge of the other species prevent them from diffusing a long distance across chloroplast envelopes. Intracellular $\mathrm{H}_{2} \mathrm{O}_{2}$ signaling engages both compartment-specific and non-specific pathways. In the case of retrograde signaling, chloroplastic $\mathrm{H}_{2} \mathrm{O}_{2}$ produced by ectopic overexpression of glycolate oxidase (GO) in chloroplasts (Fahnenstich et al., 2008) (Figure 1B) induces transcriptional changes in the nucleus, which partly, but not fully, overlap with the responses to peroxisomal $\mathrm{H}_{2} \mathrm{O}_{2}$ (Balazadeh et al., 2012; Sewelam et al., 2014).

Though ${ }^{1} \mathrm{O}_{2}$ may not move far, it can give rise to secondary messengers by reacting with nearby molecules such as $\beta$-carotene (Ramel et al., 2013). Oxidation of $\beta$-carotene produces $\beta$-cyclocitral, a reactive electrophile species that can modify transcription of ${ }^{1} \mathrm{O}_{2}$-responsive genes in the nucleus (Havaux, 2014). Whilst some $\beta$-carotenes are continuously oxidized and degraded in thylakoids during illumination with or without EL (Beisel et al., 2010), elevated production of ${ }^{1} \mathrm{O}_{2}$ and thus $\beta$-cyclocitral under photo-oxidative stress may trigger acclimatory responses that are distinct from ${ }^{1} \mathrm{O}_{2}$-induced cell death (op den Camp et al., 2003).

The number as well as the variety of agents implicated in chloroplast retrograde signaling have been increasing in the last years. For example, the redox state of the plastoquinone pool, different metabolites (e.g., tetrapyrroles, phosphoadenosine phosphate, and methylerythritol cyclodiphosphate) and hormones (abscisic acid, salicylic acid, and jasmonic acid) are regarded as such signaling agents to trigger long-term acclimatory adjustments (Dietz and Pfannschmidt, 2011; Sun et al., 2011; Estavillo et al., 2012; Xiao et al., 2012; BarajasLopez Jde et al., 2013; Karpinski et al., 2013; Dietz, 2015; Laloi and Havaux, 2015). To reconstruct signaling networks from individual components and pathways is a major challenge in understanding time-dependent regulation and interaction of stress response networks in plants (Dietz, 2015). Acclimation to photo-oxidative stress has been studied extensively in the context of HL or constant EL acclimation, in which plants manifest parallel enhancement of photoprotection and photosynthesis alongside other responses related to temperature and/or water stress. Highly dynamic FL, which predominantly elicits photoprotective responses (Alter et al., 2012), offers a complementary approach to investigate signals and pathways that are primarily engaged in photo-oxidative stress acclimation.

\section{Arabidopsis PLANTS THAT PRODUCE $\mathrm{H}_{2} \mathrm{O}_{2}$ IN CHLOROPLASTS ARE A MODEL TO STUDY PHOTO-OXIDATIVE STRESS ACCLIMATION AND SIGNALING}

Arabidopsis plants, in which GO is targeted to chloroplasts, generate $\mathrm{H}_{2} \mathrm{O}_{2}$ in chloroplasts under ambient $\mathrm{CO}_{2}$ concentrations (photorespiratory conditions) (Fahnenstich et al., 2008; Strand et al., 2015). Because the GO reaction depends on the substrate 


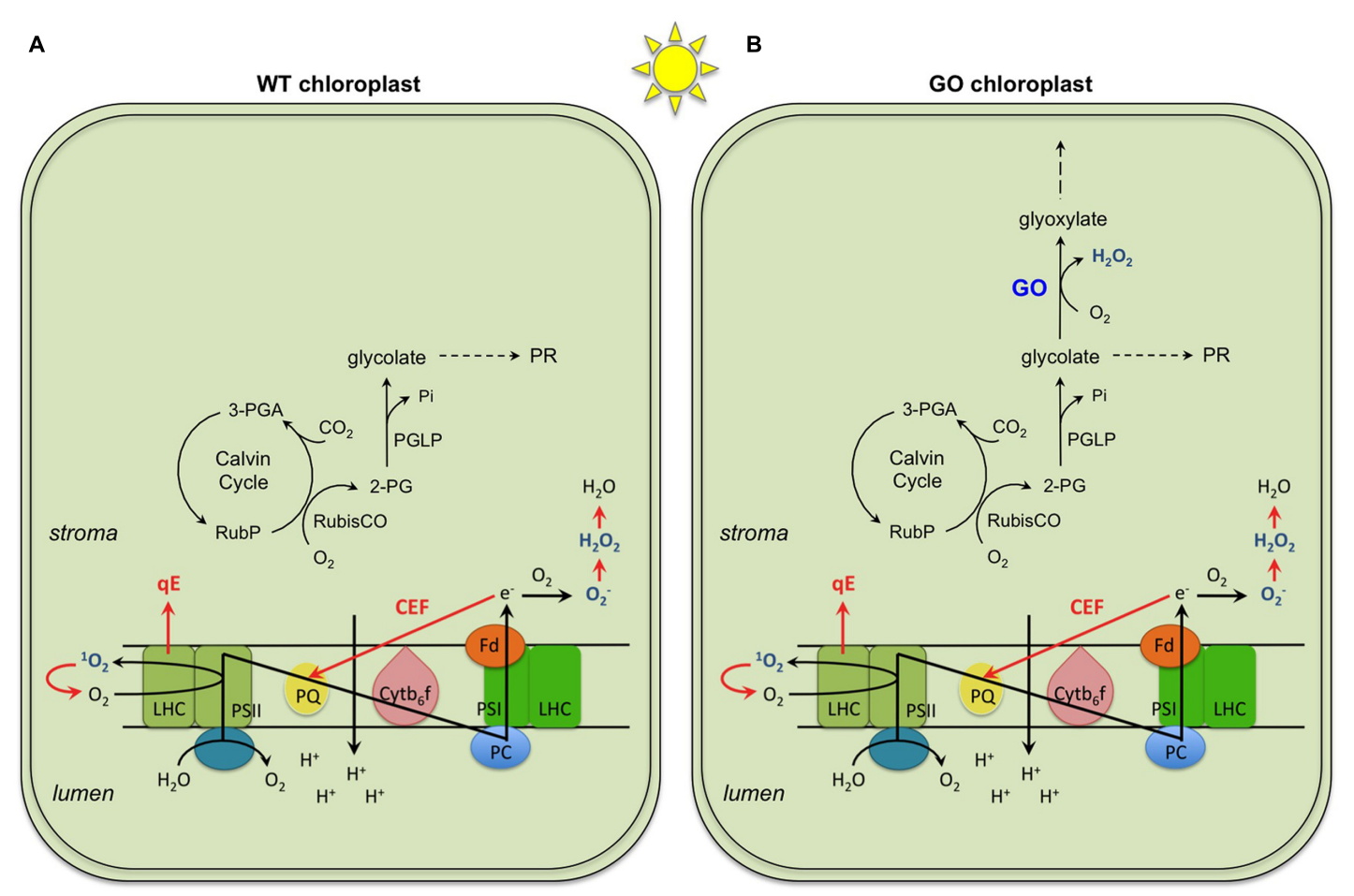

FIGURE 1 | General mechanisms of photoprotection (highlighted in red) occurring in chloroplasts of wild type (WT) (A) and the GO plants (B). Three mechanisms represented are (i) qE, the thermal energy dissipation of excess absorbed light at the light harvesting complex (LHC) of Photosystem II (PSII); (ii) CEF, the cyclic electron flow around Photosystem I (PSI), and (iii) enzymatic and non-enzymatic $\mathrm{ROS}$ scavenging through production of $\mathrm{O}_{2}$, $\mathrm{H}_{2} \mathrm{O}_{2}$ and $\mathrm{H}_{2} \mathrm{O}$. These mechanisms operate both in FL and CL; the only difference demonstrated so far is a more dramatic effect of CEF in FL than in CL. Shown in or around the thylakoid membrane are the components of photosynthetic electron transport chain: plastoquinone (PQ), cytochrome $b_{6} f c$ complex (Cytb 6 ), plastocyanin (PC), and ferredoxin (Fd). In addition, the activities of RubisCO as carboxylase and oxygenase in light and under ambient $\mathrm{CO}_{2}$ and $\mathrm{O}_{2} \mathrm{Conditions}$ are shown. In the WT, the 2-phosphoglycolate (2-PG) produced initiates the photorespiratory cycle (PR; not shown in detail in the figure; for details see (Maurino and Peterhansel, 2010)). In the GO plants, 2-PG is delivered to the PR and the glycolate produced in the chloroplasts is also used by glycolate oxidase (GO) generating $\mathrm{H}_{2} \mathrm{O}_{2}$. 3-PGA, 3-phosphoglycerate; PGLP: 2-phosphoglycolate phosphatase; RubP, ribulose 1-5, bisphosphate.

provided by the oxygenase activity of RubisCO in the light (Figure 1B), the level of GO-dependent $\mathrm{H}_{2} \mathrm{O}_{2}$ production can be controlled by changing the growth conditions. When growing in $\mathrm{LL}\left(75 \mu \mathrm{mol}\right.$ photons $\left.\mathrm{m}^{-2} \mathrm{~s}^{-1}\right)$ and ambient $\mathrm{CO}_{2}$ concentration (380 ppm), the GO plants are smaller than the wild-type (WT) plants and present patchy pale-green leaf lamina (Figure 2A; constant light, CL) as a result of $\mathrm{H}_{2} \mathrm{O}_{2}$ production in chloroplasts and overload of the antioxidant machinery (Fahnenstich et al., 2008). Under HL the GO plants develop severe oxidative lesions and ultimately bleach, whereas combinations of very LL $(30 \mu \mathrm{mol}$ photons $\mathrm{m}^{-2} \mathrm{~s}^{-1}$ ) and ambient $\mathrm{CO}_{2}$ or $\mathrm{LL}$ and high $\mathrm{CO}_{2}$ $(4,000 \mathrm{ppm})$ allow them to grow like WT (Fahnenstich et al., 2008; Balazadeh et al., 2012; Sewelam et al., 2014). Also, they become as big and green as WT and recover the WT level of photosynthetic ETR in LL when transferred from ambient to high $\mathrm{CO}_{2}$ conditions (Fahnenstich et al., 2008). These features make the GO plants a unique, well-established model to study the action of $\mathrm{H}_{2} \mathrm{O}_{2}$ in chloroplast retrograde signaling (Balazadeh et al., 2012; Sewelam et al., 2014).

Interestingly, FL conditions applied to the GO plants growing in ambient $\mathrm{CO}_{2}$-in spite of (or maybe because of) the effect of FL to impose photo-oxidative stress- provoke disappearance of the characteristic patchy pale-green phenotype of these plants (Figure 2A). This reversion to normal green leaf lamina under FL allows the GO plants to maintain similar relative growth rates in CL and FL, whereas WT exhibits growth reduction in FL (Figure 2B). The FL-induced recovery of leaf color in the GO plants is most probably due to upregulation of $\mathrm{H}_{2} \mathrm{O}_{2}$ scavenging in the chloroplast, as the patchy palegreen phenotype is a consequence of $\mathrm{H}_{2} \mathrm{O}_{2}$ accumulation (Fahnenstich et al., 2008). This assumption, i.e., FL-induced acclimatory enhancement of ROS $\left(\mathrm{H}_{2} \mathrm{O}_{2}\right)$ scavenging systems in the GO plants, is supported by the observations made in carotenogenic mutants of Arabidopsis; despite having reduced capacities for $\mathrm{qE}$ and carotenoid-dependent ROS (mainly ${ }^{1} \mathrm{O}_{2}$ ) scavenging, these mutants do not suffer from chronic photo-oxidative damage under FL conditions because they can upregulate other photoprotective and ROS scavenging mechanisms by long-term acclimation (Caliandro et al., 2013). The increased activity of superoxide dismutase (SOD) found in leaves of WT following FL acclimation (Alter et al., 2012) also points to an increased detoxification capacity for $\mathrm{H}_{2} \mathrm{O}_{2}$ which arises from disproportionation of $\mathrm{O}_{2}^{\bullet-}$ catalyzed by SOD. 

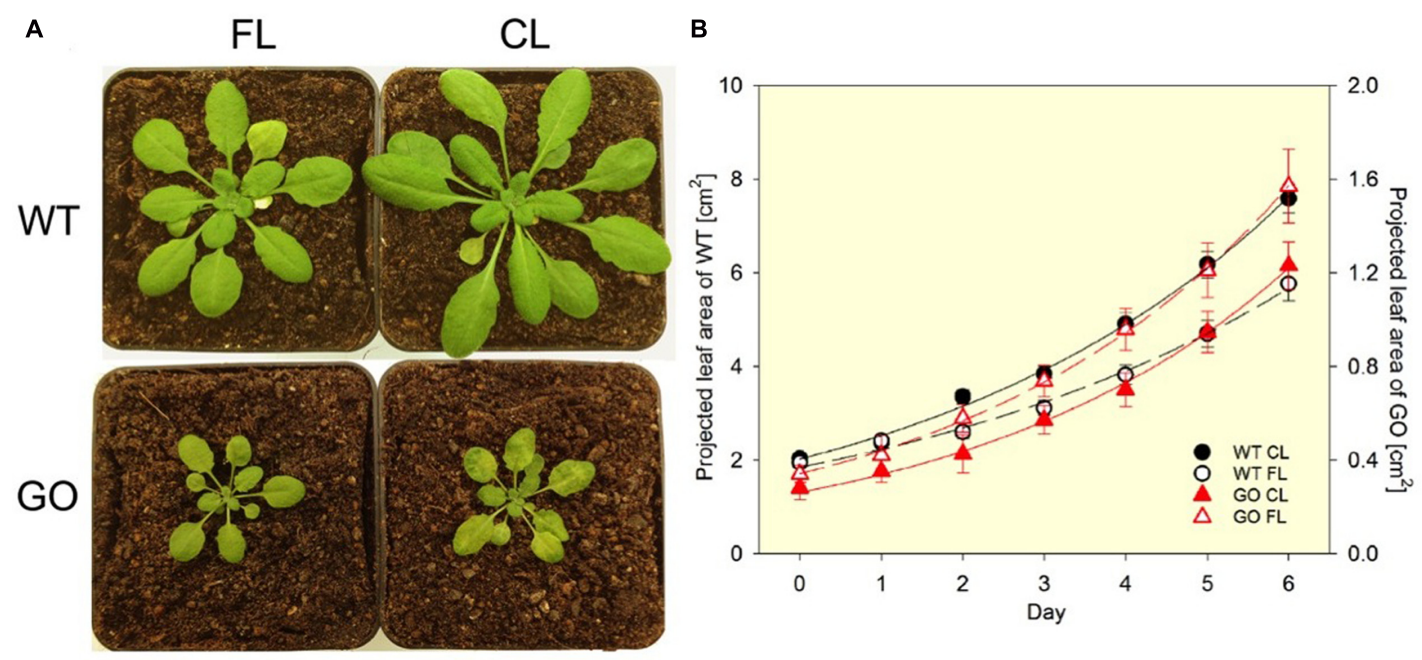

FIGURE 2 | Phenotypes of wild-type (WT) and GO plants grown under fluctuating light (FL) and constant light (CL) conditions in ambient $\mathrm{CO}_{2}$. (A) Under FL (switching between $\sim 50 \mu \mathrm{mol}$ photons $\mathrm{m}^{-2} \mathrm{~s}^{-1}$ for $280 \mathrm{~s}$ and $\sim 1000 \mu \mathrm{mol}$ photons $\mathrm{m}^{-2} \mathrm{~s}^{-1}$ for $20 \mathrm{~s}$ ) the GO plants have normal green leaves, while under $\mathrm{CL}\left(\sim 75 \mu \mathrm{mol}\right.$ photons $\left.\mathrm{m}^{-2} \mathrm{~s}^{-1}\right)$ they develop pale-green patches due to the chloroplastic production of $\mathrm{H}_{2} \mathrm{O}_{2}$. The pictures were taken after 8-days exposure to FL or CL. (B) The FL condition reduces leaf growth of the WT plants (left $y$-axis). The GO plants (right $y$-axis) are much smaller than the WT and the exposure to FL does not impair growth in the GO plants compared to the $\mathrm{CL}$ condition. Projected leaf area is the leaf area that is visible in a 2D top-view image. Solid and broken lines show exponential regression for the $\mathrm{CL}$ and FL data sets, respectively. The $R^{2}$ values of the fitting are: 0.996 (WT CL), 0.991 (WT FL), 0.997 (GO $\mathrm{CL})$, and 0.999 (GO FL). Relative growth rates (\% $\mathrm{d}^{-1}$ ) obtained by the regression are: $22.1 \pm 0.6(\mathrm{WT} \mathrm{CL}), 18.7 \pm 0.8(\mathrm{WT} \mathrm{FL}), 25.7 \pm 0.7$ (GO CL), and 25.4 \pm 0.3 (GO FL). The difference in relative growth rate between $\mathrm{CL}$ and $\mathrm{FL}$ is significant for the $\mathrm{WT}(P=0.002) . n=16(\mathrm{WT})$ and $9(\mathrm{GO})$.

Thus, we hypothesize that long-term acclimatory upregulation of $\mathrm{H}_{2} \mathrm{O}_{2}$ scavenging occurs in chloroplasts under FL conditions. $\mathrm{H}_{2} \mathrm{O}_{2}$ reduction in chloroplasts could proceed mainly via peroxidase systems, including glutathione peroxidase (Gpx) and peroxiredoxin ( $\operatorname{Prx})$ in the stroma, as well as thylakoidbound and stromal ascorbate peroxidase (tAPX, sAPX) coupled to monodehydroascorbate reductase (MDHAR) and also dehydroascorbate reductase (DHAR) (Foyer and Noctor, 2011). Gpx and Prx use thiol-based peroxide-detoxification mechanisms that are maintained by glutathione and glutathione reductase (GR) (Dietz and Pfannschmidt, 2011). Regeneration of ascorbate by DHAR is also dependent on glutathione and GR, while MDHAR uses $\mathrm{NAD}(\mathrm{P}) \mathrm{H}$ to regenerate ascorbate. In addition to the removal of $\mathrm{H}_{2} \mathrm{O}_{2}$, other photoprotective mechanisms such as CEF and carotenoid-dependent reactions could also contribute to the rescuing of the GO phenotype in FL by keeping the level of ROS production under control (Figure 1). Indeed, $\mathrm{CEF}$ is activated by chloroplastic $\mathrm{H}_{2} \mathrm{O}_{2}$ produced in HL (Strand et al., 2015) and carotenoid contents (especially xanthophyll-cycle pigments) increase in leaves exposed to FL (Alter et al., 2012; Caliandro et al., 2013).

\section{FUTURE DIRECTIONS}

So far it is not known whether, and if yes, which $\mathrm{H}_{2} \mathrm{O}_{2}$ scavenging pathways are upregulated in chloroplasts during $\mathrm{FL}$ acclimation. This could be studied by analyzing antioxidant defense systems in the GO plants during acclimation to $\mathrm{CL}$ and FL conditions. An important question that can then be tackled is the long-term acclimation of $\mathrm{H}_{2} \mathrm{O}_{2}$ scavenging systems. Signal agents, which are generated in FL conditions and lead to acclimatory enhancement of $\mathrm{H}_{2} \mathrm{O}_{2}$ scavenging, must be different from the signals induced by chloroplastic $\mathrm{H}_{2} \mathrm{O}_{2}$ produced in the GO plants under $\mathrm{CL}$ and ambient $\mathrm{CO}_{2}$ levels. Close inspections of the GO plants during FL acclimation at different response levels - from gene expression, protein and metabolite accumulation to physiological phenotype- could shed light on components and signal agents involved in upregulation of $\mathrm{H}_{2} \mathrm{O}_{2}$ scavenging under photo-oxidative stress. Once candidate molecules are identified, the unique feature of the GO plants, which visualizes acclimatory changes in $\mathrm{H}_{2} \mathrm{O}_{2}$ metabolism under FL, can be exploited again as the genetic background to assess the efficacy of those molecules in upregulating $\mathrm{H}_{2} \mathrm{O}_{2}$ detoxification. The nature of chloroplast retrograde signaling in FL (dynamic EL), as compared with that in HL (constant EL), inspires further experiments and investigations.

\section{AUTHOR CONTRIBUTIONS}

SM and VM contributed equally to writing the manuscript. TS performed the growth analysis shown in Figure 2.

\section{FUNDING}

This work was supported by the Deutsche Forschungsgemeinschaft. 


\section{REFERENCES}

Alter, P., Dreissen, A., Luo, F. L., and Matsubara, S. (2012). Acclimatory responses of Arabidopsis to fluctuating light environment: comparison of different sunfleck regimes and accessions. Photosynth. Res. 113, 221-237. doi: 10.1007/s11120-012-9757-2

Apel, K., and Hirt, H. (2004). Reactive oxygen species: metabolism, oxidative stress, and signal transduction. Annu. Rev. Plant Biol. 55, 373-399. doi: 10.1146/annurev.arplant.55.031903.141701

Asada, K. (1999). The water-water cycle in chloroplasts: scavenging of active oxygens and dissipation of excess photons. Annu. Rev. Plant Physiol. Plant Mol. Biol. 50, 601-639. doi: 10.1146/annurev.arplant.50.1.601

Balazadeh, S., Jaspert, N., Arif, M., Mueller-Roeber, B., and Maurino, V. G. (2012). Expression of ROS-responsive genes and transcription factors after metabolic formation of $\mathrm{H}_{2} \mathrm{O}_{2}$ in chloroplasts. Front. Plant Sci. 3:234. doi: 10.3389/fpls.2012.00234

Barajas-Lopez Jde, D., Blanco, N. E., and Strand, A. (2013). Plastid-to-nucleus communication, signals controlling the running of the plant cell. Biochim. Biophys. Acta 1833, 425-437. doi: 10.1016/j.bbamcr.2012.06.020

Beisel, K. G., Jahnke, S., Hofmann, D., Koppchen, S., Schurr, U., and Matsubara, S. (2010). Continuous turnover of carotenes and chlorophyll a in mature leaves of Arabidopsis revealed by $14 \mathrm{CO} 2$ pulse-chase labeling. Plant Physiol. 152, 2188-2199. doi: 10.1104/pp.109.151647

Caliandro, R., Nagel, K. A., Kastenholz, B., Bassi, R., Li, Z., Niyogi, K. K., et al. (2013). Effects of altered alpha- and beta-branch carotenoid biosynthesis on photoprotection and whole-plant acclimation of Arabidopsis to photo-oxidative stress. Plant Cell Environ. 36, 438-453. doi: 10.1111/j.1365-3040.2012.02586.x

Dietz, K. J. (2015). Efficient high light acclimation involves rapid processes at multiple mechanistic levels. J. Exp. Bot. 66, 2401-2414. doi: 10.1093/jxb/eru505

Dietz, K. J., and Pfannschmidt, T. (2011). Novel regulators in photosynthetic redox control of plant metabolism and gene expression. Plant Physiol. 155, 1477-1485. doi: 10.1104/pp.110.170043

Dietz, K. J., Turkan, I., and Krieger-Liszkay, A. (2016). Redox- and reactive oxygen species-dependent signaling into and out of the photosynthesizing chloroplast. Plant Physiol. 171, 1541-1550. doi: 10.1104/pp.16.00375

Endo, T., and Asada, K. (2008). "Photosystem I and photoprotection: cycling electron flow and water-water cycle," in Photoprotection, Photoinhibition, Gene Regulation, and Environment, eds B. Demmig-Adams, W. W. Adams, and A. K. Wattoo (Berlin: Springer), 205-211.

Estavillo, G. M., Chan, K. X., Phua, S. Y., and Pogson, B. J. (2012). Reconsidering the nature and mode of action of metabolite retrograde signals from the chloroplast. Front. Plant Sci. 3:300. doi: 10.3389/fpls.2012.00300

Fahnenstich, H., Scarpeci, T. E., Valle, E. M., Flugge, U. I., and Maurino, V. G. (2008). Generation of hydrogen peroxide in chloroplasts of Arabidopsis overexpressing glycolate oxidase as an inducible system to study oxidative stress. Plant Physiol. 148, 719-729. doi: 10.1104/pp.108.126789

Foyer, C. H., and Noctor, G. (2000). Oxygen processing in photosynthesis: regulation and signalling. New Phytol. 146, 359-388. doi: 10.1046/j.14698137.2000.00667.x

Foyer, C. H., and Noctor, G. (2011). Ascorbate and glutathione: the heart of the redox hub. Plant Physiol. 155, 2-18. doi: 10.1104/pp.110.167569

Havaux, M. (2014). Carotenoid oxidation products as stress signals in plants. Plant J. 79, 597-606. doi: 10.1111/tpj.12386

Karpinski, S., Szechynska-Hebda, M., Wituszynska, W., and Burdiak, P. (2013). Light acclimation, retrograde signalling, cell death and immune defences in plants. Plant Cell Environ. 36, 736-744. doi: 10.1111/pce.12018

Kirschbaum, M. U., and Pearcy, R. W. (1988). Gas exchange analysis of the relative importance of stomatal and biochemical factors in photosynthetic induction in Alocasia macrorrhiza. Plant Physiol. 86, 782-785. doi: 10.1104/pp. 86.3 .782
Laloi, C., and Havaux, M. (2015). Key players of singlet oxygen-induced cell death in plants. Front. Plant Sci. 6:39. doi: 10.3389/fpls.2015. 00039

Leakey, A. D. B., Press, M. C., Scholes, J. D., and Watling, J. R. (2002). Relative enhancement of photosynthesis and growth at elevated $\mathrm{CO} 2$ is greater under sunflecks than uniform irradiance in a tropical rain forest tree seedling. Plant Cell Environ. 25, 1701-1714. doi: 10.1046/j.1365-3040.2002. 00944.x

Li, Z., Wakao, S., Fischer, B. B., and Niyogi, K. K. (2009). Sensing and responding to excess light. Annu. Rev. Plant Biol. 60, 239-260. doi: 10.1146/annurev.arplant.58.032806.103844

Maurino, V. G., and Peterhansel, C. (2010). Photorespiration: current status and approaches for metabolic engineering. Curr. Opin. Plant Biol. 13, 249-256. doi: 10.1016/j.pbi.2010.01.006

Mittler, R., Vanderauwera, S., Suzuki, N., Miller, G., Tognetti, V. B., Vandepoele, K., et al. (2011). ROS signaling: the new wave? Trends Plant Sci. 16, 300-309. doi: 10.1016/j.tplants.2011.03.007

op den Camp, R. G., Przybyla, D., Ochsenbein, C., Laloi, C., Kim, C., Danon, A., et al. (2003). Rapid induction of distinct stress responses after the release of singlet oxygen in Arabidopsis. Plant Cell 15, 2320-2332. doi: 10.1105/tpc.014662

Pearcy, R. W. (1990). Sunflecks and photosynthesis in plant canopies. Annu. Rev. Plant Physiol. Plant Mol. Biol. 41, 421-453. doi: 10.1146/annurev.pp.41.060190.002225

Ramel, F., Mialoundama, A. S., and Havaux, M. (2013). Nonenzymic carotenoid oxidation and photooxidative stress signalling in plants. J. Exp. Bot. 64, 799805. doi: $10.1093 / \mathrm{jxb} / \mathrm{ers} 223$

Retkute, R., Smith-Unna, S. E., Smith, R. W., Burgess, A. J., Jensen, O. E., Johnson, G. N., et al. (2015). Exploiting heterogeneous environments: does photosynthetic acclimation optimize carbon gain in fluctuating light? J. Exp. Bot. 66, 2437-2447. doi: 10.1093/jxb/erv055

Sewelam, N., Jaspert, N., Van der Kelen, K., Tognetti, V. B., Schmitz, J., Frerigmann, H., et al. (2014). Spatial $\mathrm{H} 2 \mathrm{O} 2$ signaling specificity: $\mathrm{H} 2 \mathrm{O} 2$ from chloroplasts and peroxisomes modulates the plant transcriptome differentially. Mol. Plant 7, 1191-1210. doi: 10.1093/mp/ssu070

Strand, D. D., Livingston, A. K., Satoh-Cruz, M., Froehlich, J. E., Maurino, V. G., and Kramer, D. M. (2015). Activation of cyclic electron flow by hydrogen peroxide in vivo. Proc. Natl. Acad. Sci. U.S.A. 112, 5539-5544. doi: 10.1073/pnas.1418223112

Sun, X., Feng, P., Xu, X., Guo, H., Ma, J., Chi, W., et al. (2011). A chloroplast envelope-bound PHD transcription factor mediates chloroplast signals to the nucleus. Nat. Commun. 2:477. doi: 10.1038/ ncomms1486

Xiao, Y., Savchenko, T., Baidoo, E. E., Chehab, W. E., Hayden, D. M., Tolstikov, V., et al. (2012). Retrograde signaling by the plastidial metabolite MEcPP regulates expression of nuclear stress-response genes. Cell 149, 1525-1535. doi: 10.1016/j.cell.2012.04.038

Yin, Z. H., and Johnson, G. N. (2000). Photosynthetic acclimation of higher plants to growth in fluctuating light environments. Photosynth. Res. 63, 97-107. doi: 10.1023/A:1006303611365

Conflict of Interest Statement: The authors declare that the research was conducted in the absence of any commercial or financial relationships that could be construed as a potential conflict of interest.

Copyright $\odot 2016$ Matsubara, Schneider and Maurino. This is an open-access article distributed under the terms of the Creative Commons Attribution License (CC BY). The use, distribution or reproduction in other forums is permitted, provided the original author(s) or licensor are credited and that the original publication in this journal is cited, in accordance with accepted academic practice. No use, distribution or reproduction is permitted which does not comply with these terms. 